\title{
19. Wohnung und Wohnkosten im Alter
}

\author{
Sonja Nowossadeck \& Heribert Engstler
}

\section{Kernaussagen}

Der Anteil der 40- bis 85-Jährigen, die in der eigenen Immobilie wohnen, hat sich zwischen 1996 und 2014 erhöht: Bei den 70- bis 85-Jährigen ist die Wohneigentümerquote in dieser Zeit überdurchschnittlich von 47,9 Prozent auf 59,7 Prozent gestiegen. Zugenommen hat allerdings auch der Anteil der Personen mit Wohneigentum, die in diesem Alter noch ein Immobiliendarlehen abzahlen müssen. Die Wohneigentümerquote ist insgesamt in Ostdeutschland zwar immer noch niedriger als in Westdeutschland, der Unterschied zwischen Ost und West hat sich bei den 40- bis 85-Jährigen jedoch zwischen 1996 und 2014 halbiert (von 26,9 auf 12,7 Prozentpunkte).

Die eigene Wohnsituation wird im Jahr 2014 von einer großen Mehrheit der 40- bis 85-Jährigen als gut bewertet: 89,7 Prozent der 40- bis 85-Jährigen bewerten im Jahr 2014 ihre Wohnsituation als gut. Von 1996 bis 2014 hat sich in Ostdeutschland die Bewertung der Wohnsituation deutlich verbessert und sich damit dem westdeutschen Niveau angeglichen. Im Jahr 2014 bewerten die Ältesten (70 bis 85 Jahre) mit 92,0 Prozent ihre Wohnsituation im Vergleich zu den 40- bis 54-Jährigen (88,0 Prozent) und den 55- bis 69-Jährigen (90,1 Prozent) häufiger als gut. Auch in einfachen Wohnlagen bewerten die Bewohnerinnen und Bewohner ihre Wohnsituation mehrheitlich als gut $(68,1$ Prozent), wenn auch weniger häufig als in gehobenen (96,2 Prozent) und mittleren Wohnlagen (87,9 Prozent).

Die monatliche Wohnkostenbelastung in Prozent des Einkommens hat zwischen 1996 und 2014 zugenommen: Der Anteil des Haushaltseinkommens, den Mieterinnen und Mieter für die Miete und die Nebenkosten ausgeben, ist zwischen 1996 und 2014 von 27,9 auf 35,1 Prozent gestiegen. Besonders hoch ist die Mietbelastung für ältere alleinlebende Frauen (45,2 Prozent). Eine relativ niedrige Wohnkostenbelastung haben Haushalte, die im schuldenfreien Wohneigentum leben (16,0 Prozent).

Barrierereduzierte Wohnungen sind im Jahr 2014 wenig verbreitet: Unter den 40- bis 85-Jährigen leben im Jahr 2014 nur 2,9 Prozent in einer barrierereduzierten Wohnung. Von den Personen, die eine Gehhilfe, einen Rollator beziehungsweise einen Rollstuhl benutzen, haben im Jahr 2014 6,9 Prozent eine barrierereduzierte Wohnung.

\subsection{Einleitung}

Die meisten Menschen wohnen bis ins hohe Alter in einer privaten Wohnung. 97 Prozent der 60-Jährigen und Älteren und immer noch 90 Prozent der 80-Jährigen und Älteren leben nach Angaben des Mikrozensus 2014 in einem privaten Haushalt, also mit Partnerin oder Partner, allein oder mit anderen Verwandten in einer Wohnung oder einem Haus (Statistisches Bundesamt 2015). Selbst wenn Ältere pflegebedürftig werden, bleibt für die Mehrzahl von ihnen die eigene Wohnung der zentrale Lebensort. Ältere verbringen viel Zeit in der Wohnung, mehr als 
jüngere Menschen, die ihr Wohnumfeld für Alltagsaktivitäten häufig für längere Zeit verlassen (Engstler, Menning, Hoffmann, \& Tesch-Römer 2004). Mit steigendem Alter und zunehmenden Gesundheitsproblemen neigen ältere Menschen dazu, ihren Aktionsradius zu reduzieren und sich mehr in der Wohnung und dem unmittelbaren Wohnumfeld aufzuhalten. Gleichzeitig steigt ihre Vulnerabilität gegenüber Mängeln in der Wohnung und der Wohnumgebung. Die Wohnung mit ihrem Wohnumfeld bestimmt daher für Ältere zu einem wesentlichen Teil, wie selbstbestimmt sie altern (Kocka \& Staudinger 2009; Oswald, Jopp, Rott, \& Wahl 2011).

Der Deutsche Alterssurvey (DEAS) enthält Informationen zu verschiedenen Aspekten des Wohnens, von den Wohnformen und der subjektiven Bewertung der Wohnsituation bis zur Wohnungsausstattung und den Wohnkosten. Dies ermöglicht es, verschiedene Facetten des Wohnens zu beleuchten. Dieses Kapitel widmet sich daher mehreren Aspekten des Stands und der Entwicklung der Wohnsituation der Menschen in der zweiten Lebenshälfte.

Ein erster Aspekt des Kapitels ist das Wohnen in Mietwohnungen und im Wohneigentum. Aus historischen Gründen ist die Wohneigentumsquote in Deutschland im europäischen Vergleich eher niedrig (Kaltenbrunner \& Waltersbacher 2014). In den ersten Jahrzehnten des Wiederaufbaus nach dem Zweiten Weltkrieg stand in beiden Teilen Deutschlands die Schaffung günstigen Wohnraums durch den Mietwohnungsbau im Vordergrund. Während in Westdeutschland anschließend auch die Bildung von Wohneigentum an Bedeutung gewann, kam dem privaten Wohneigentum in Ostdeutschland bis zur Wende nur eine untergeordnete Bedeutung zu. Dies hat sich nach der Wiedervereinigung geändert. Die Zuwachsrate des Wohneigentums ist in den neuen Bundesländern mittlerweile höher als in den alten Bundesländern (ebd.). Untersucht wird im vorliegenden Beitrag, wie weit sich die Ost-West-Unterschiede in der Wohneigentümerquote verringert haben und in welchem Maße die einzelnen Altersgruppen am Trend zu mehr Wohneigentum teilhaben. Erwartet wird ein überdurchschnittlicher Anstieg bei den über 50-Jährigen, die nun häufi- ger mit selbst erworbenem oder von der älteren Generation übertragenem Wohneigentum ins Alter kommen.

Die Wohnung ist der zentrale Lebensort im Alter, daher stellt die Bewertung der Wohnsituation einen wichtigen Indikator für die Lebensqualität in dieser Lebensphase dar. Noch zu Beginn der 1990er Jahre unterschied sich die Wohnqualität in West- und Ostdeutschland deutlich voneinander und dementsprechend war auch die Bewertung der Wohnsituation in Ostdeutschland weniger positiv als in Westdeutschland (Motel-Klingebiel, Künemund, \& Bode 2005). Inzwischen haben sich die Wohnverhältnisse zwischen West und Ost angenähert und dementsprechend ist auch eine ähnliche Bewertung der Wohnsituation in beiden Landesteilen zu erwarten. Das ist auch aus den Befunden von Priem und Schupp (2014) abzuleiten, die feststellen, dass sich die Ost-West-Lücke unter anderem in der Zufriedenheit mit der Wohnung endgültig geschlossen hat, da sich die Wohnstandards angeglichen haben.

Als weiterer Aspekt des Wohnens im Alter werden die Wohnkosten von Mieter- und Eigentümerhaushalten näher betrachtet. Im Allgemeinen sind die Mietbeträge bei Seniorenhaushalten etwas niedriger als bei jüngeren Altersgruppen. Allerdings ist die Mietbelastung als Anteil am verfügbaren Einkommen, der für die Wohnkosten aufgewendet wird, bei Personen im Ruhestand überdurchschnittlich hoch, insbesondere bei Alleinstehenden (Frick \& Droß 2011; Statistisches Bundesamt 2012). Zudem hat auf dem Wohnungsmarkt das Angebot günstiger Mietwohnungen abgenommen und für viele hält die Einkommensentwicklung nicht mit der Wohnkostenentwicklung Schritt (Pestel Institut 2012; BertelsmannStiftung 2013). Der Erwerb von Wohneigentum gilt als eine Strategie der Altersvorsorge mit der Idee, dadurch im Alter Wohnkosten einzusparen. Jedoch ist unklar, wem es möglich ist, im Alter im schuldenfreien Wohneigentum zu wohnen und wie hoch die Einsparungen der monatlichen Wohnkosten bei Wohneigentümern tatsächlich sind. Vor diesem Hintergrund wird zunächst beschrieben, wie sich der Anteil der Wohneigentümer in der zweiten Lebenshälfte verändert hat. Außerdem 
wird untersucht, wie sich die Wohnkostenbelastung der Älteren gewandelt hat und welche sozialen Gruppen besonders stark belastet sind.

Die demografische Alterung erfordert in großem Umfang eine den Bedürfnissen Älterer angepasste Wohnungsausstattung. Barrierefreiheit ist nicht für die Wohnungen aller älteren Menschen notwendig, sie sollte jedoch zumindest für mobilitätseingeschränkte ältere Menschen zur Verfügung stehen, um auch ihnen eine selbstständige Lebensführung zu ermöglichen. Eine Studie des Kuratoriums Deutsche Altershilfe (KDA) schätzt, dass etwa fünf Prozent aller Seniorenhaushalte (65 Jahre und älter) in einer weitgehend barrierefreien Wohnung leben (Bundesministerium für Verkehr Bau und Stadtentwicklung (BMVBS) 2011; Kuratorium Deutsche Altershilfe \& Wüstenrot Stiftung 2014). Als Mindestanforderungen einer weitgehend barrierefreien Wohneinheit werden folgende Kriterien genannt, die (zum Teil in abgekürzter Form) auch im DEAS abgefragt werden: nicht mehr als drei Stufen zum Haus oder zum Wohnungseingang (ohne technische Hilfen zur Überwindung der Barrieren), keine Stufen innerhalb der Wohnung (ohne technische Hilfen zur Überwindung der Barrieren), ausreichende Bewegungsflächen und Türbreiten im Sanitärbereich sowie das Vorhandensein einer bodengleichen Dusche. Das Festlegen dieser Ausstattungskriterien der Wohnung führt zu den oben genannten, vergleichsweise niedrigen Anteilen barrierereduzierter Wohnungen. Eine weitere Studie auf Basis des Sozio-oekonomischen Panels (SOEP) erfasste subjektive Einschätzungen von als altengerecht empfundenen Wohnungen und erhielt auf dieser Basis deutlich höhere Anteile: 19,6 Prozent aller Befragten, 29,9 Prozent der Befragten mit mindestens einer über 65-jährigen Person im Haushalt und 48,8 Prozent der Befragten mit mindestens einer pflegebedürftigen Person im Haushalt schätzten ihre Wohnungsausstattung als altengerecht und barrierefrei ein (Rothgang, Kalwitzki, Müller, Runte, \& Unger 2015). Diese Unterschiede zwischen objektivierten und subjektiven Einschätzungen zeigen, dass Ältere auch beim Wohnen erhebliche Anpassungsleistungen an die gegebenen Umstände erbringen und dass die Barrierefreiheit der Wohnung nicht immer das entscheidende Kriterium für die Passfähigkeit der Wohnung ist. Die in diesem Kapitel ausgewiesenen Anteilswerte zu barrierereduzierten Wohnungen lehnen sich an das Vorgehen der KDA-Studie an, um einen Befund zum tatsächlichen Umfang barrierereduzierten Wohnens in der zweiten Lebenshälfte zu erhalten. Es wird untersucht, welche Kriterien barrierereduzierten Wohnens die Wohnungen älterer Menschen in Deutschland derzeit erfüllen und welche Unterschiede in der Versorgung mit diesen Wohnungen existieren. Die Wohnbedingungen von Älteren mit einer eingeschränkten Mobilität bilden einen weiteren Analyseschwerpunkt, da diese Gruppe in besonderem Maße auf eine Barrierefreiheit der Wohnung angewiesen ist.

Im Einzelnen werden im vorliegenden Kapitel die folgenden Fragen untersucht:

1. Steigt der Anteil der Personen, die im eigenen Wohneigentum leben? Hat Ostdeutschland beim Besitz von Wohneigentum aufgeholt?

2. Wie bewerten Ältere ihre Wohnsituation und hat sich das über die Jahre verändert?

3. Wie hat sich die Wohnkostenbelastung über die Jahre bei Mieterinnen und Mietern sowie bei Personen mit Wohneigentum verändert? Sind Ältere durch Wohnkosten insgesamt stärker belastet als andere Altersgruppen? Welche Kostenentlastung bringt das Wohnen in der eigenen Immobilie im Alter?

4. Wie barrierereduziert sind die Wohnungen der Älteren? Wie sind die Wohnungen von Menschen mit starken funktionalen Einschränkungen beziehungsweise mit Hilfsmittelabhängigkeit (Gehhilfe, Rollator, Rollstuhl oder ähnliches) ausgestattet? 


\subsection{Datengrundlage ${ }^{1}$}

Daten. Die Auswertungen und Ergebnisse beruhen auf den Daten der vier Basisstichproben des DEAS der Erhebungsjahre 1996, 2002, 2008 und 2014 (vgl. Kapitel 2). Es werden jeweils deskriptive Befunde zur Entwicklung und Verteilung folgender Merkmale dargestellt:

Wohneigentum. Aus der für alle vier Erhebungsjahre aus dem mündlichen Interview vorliegenden Angabe darüber, ob man als Eigentümerin/Eigentümer, Hauptmieterin/Hauptmieter ist oder zur Untermiete wohnt oder einen anderen Bewohnerstatus hat (mietfrei, Wohnrecht etc.), wird der Anteil der Personen mit Wohneigentum berechnet. Abhängig davon, ob für das Wohneigentum noch Raten eines Immobiliendarlehens zu bezahlen sind, wird zwischen Wohneigentümerinnen und -eigentümern mit und ohne Immobiliendarlehen unterschieden.

Bewertung der Wohnsituation. Die Bewertung der Wohnsituation wurde mit einer fünfteiligen Skala erfragt. („Einmal insgesamt betrachtet, wie bewerten Sie Ihre derzeitige Wohnsituation?" $1=$,sehr gut $/ 2=$, gut $/ 3=$, mittel $/$ $4=$,schlecht' $/ 5=$,sehr schlecht' $)$. Für die Analyse wurden diese Angaben in eine dichotome Variable rekodiert: , gut ${ }^{\text { }}=$ Antwortkategorien 1 und 2,, $\mathrm{mittel} / \mathrm{schlecht}^{ }=$Antwortkategorien 3, 4 und 5.

Wohnkosten. Bei zur Miete Wohnenden umfassen die Wohnkosten die monatliche Miete einschließlich aller Nebenkosten. Diese Bruttowarmmiete wird in jedem Erhebungsjahr im mündlichen Interview als Gesamtbetrag erhoben, einschließlich einer Bestätigung, dass es sich dabei um die Miete inklusive aller Nebenkosten handelt. Bei Wohneigentümerinnen und Wohneigentümern werden die monatlichen Kosten, die ihr Haushalt für Strom, Gas, Wasser, Heizung, Müllabfuhr und sonstige Nebenkosten aufwendet, gesondert als Summenbetrag erhoben. Sofern für das selbstbewohnte Wohneigentum noch Abzahlungen für ein Immobi-

1 Die Daten des DEAS können für wissenschaftliche Zwecke kostenlos beim Forschungsdatenzentrum des DZA (www.fdz-dza.de) bezogen werden. liendarlehen geleistet werden, ist die Höhe der monatlichen beziehungsweise. auf den Monat bezogenen Zahlungsrate anzugeben. Diese wird den Wohnkosten hinzu gerechnet. Falls bei den Angaben zu den Wohnkosten keine genauen Beträge angegeben werden können, ist der ungefähre Betrag zu nennen. Im Beitrag werden die monatlichen Wohnkosten der Hauptmieterinnen und -mieter und Personen mit Wohneigentum ohne und mit Immobiliendarlehen berichtet.

Wohnkostenbelastung. Die Wohnkostenbelastung setzt die monatlichen Wohnkosten in Relation zum monatlichen Nettoeinkommen des Haushalts. Sie gibt an, wieviel Prozent des Einkommens für die Wohnkosten ausgegeben werden.

Barrierereduzierte Wohnung. Die Kriterien für Barrierefreiheit in Wohnungen werden in der Norm DIN 18040-2 sehr detailliert und umfangreich geregelt. Das betrifft zum Beispiel die umfangreichen Regeln zur Größe von Bewegungsflächen, zur baulichen Badausstattung bis hin zum zulässigen Durchmesser, den Handläufe haben dürfen. Die Gesamtheit dieser Kriterien kann in einem Survey nicht abgefragt werden. In der Befragung 2014 wurde im DEAS erstmals versucht, die Vielzahl von Kriterien der Barrierefreiheit auf einige wesentliche und für die Befragten bewertbare Indikatoren zu reduzieren. Im Einzelnen wurden im schriftlichen Fragebogen das Vorhandensein oder Fehlen folgender Merkmale erfasst (leicht verkürzte Darstellung): Wohnung beziehungsweise Haus ist stufenlos erreichbar, alle Treppen haben auf beiden Seiten einen Handlauf, alle Zimmertüren haben keine oder nur niedrige Schwellen, Türen der Sanitärräume sind breit genug für Gehhilfe/Rollator (mindestens $80 \mathrm{~cm}$ ), Türen der Sanitärräume gehen nach außen auf, innerhalb der Wohnung sind alle Zimmer stufenlos erreichbar.

Die Übertragung dieser Kriterien auf einen Indikator für eine barrierereduzierte Wohnung erfolgt in Anlehnung an das Vorgehen einer KDA-Repräsentativbefragung von Senioren- 
haushalten (Bundesministerium für Verkehr Bau und Stadtentwicklung (BMVBS) 2011). Es werden zwei Arten von Barrieren definiert: Barrieren beim Zugang zur Wohnung und Barrieren in der Wohnung. Ein barrierereduzierter Zugang zur Wohnung liegt in unseren Analysen vor, wenn die Wohnung beziehungsweise das Haus stufenlos erreichbar ist. Eine Wohnung ist innen barrierereduziert, wenn die Zimmertüren keine oder nur niedrige Türschwellen haben, wenn die Türen von Bad und WC breit genug sind für Gehhilfen/Rollator (mindestens $80 \mathrm{~cm}$ ) und außerdem nach außen öffnen und wenn alle Zimmer in der Wohnung stufenlos erreichbar sind. Von einer barrierereduzierten Wohnung sprechen wir, wenn nach dieser Definition sowohl ein barrierereduzierter Zugang zur Wohnung vorhanden ist und die Wohnung auch im Inneren barrierereduziert ist. Die Badausstattung (bodengleiche Dusche, Dusche mit Duschtasse, Badewanne) wurde nicht in die Bewertung einbezogen. Jede funktionale Einschränkung der Bewohnerinnen und Bewohner braucht eigene Badlösungen, eine Wanne mit Wannenlift kann unter Umständen funktionaler sein als eine bodengleiche Dusche, die zu eng ist für einen Rollstuhl.

Gruppierungsvariablen. Es werden Geschlechtsunterschiede (männlich/weiblich), Altersgruppendifferenzen ( 40 bis 54 Jahre, 55 bis 69 Jahre, 70 bis 85 Jahre) und regionale Unterschiede zwischen West- und Ostdeutschland untersucht. Westdeutschland umfasst die alten Bundesländer einschließlich dem früheren Westteil Berlins; Ostdeutschland umfasst die neuen Bundesländer inklusive dem früheren Ostteil Berlins. Bei der Betrachtung der Wohnkosten und der subjektiven Bewertung der Wohnsituation werden bivariat auch Unterschiede nach der Wohnlage untersucht. Die von den Interviewerinnen und Interviewern vorgenommene Einschätzung der Wohnlage auf einer fünfstufigen Skala wird dabei rekodiert zu den drei Kategorien, einfache (1-2), ,mittlere` (3) und ,gehobene ' Wohnlage (4-5).

Daneben werden Mobilitätseinschränkungen der befragten Person und der siedlungsstrukturelle Kreistyp des Wohnkreises der Befragten als Gruppierungsvariable genutzt:

Mobilitätseinschränkungen. Einschränkungen in der Mobilität werden über die im schriftlichen Fragebogen erhobene Nutzung eines Mobilitätshilfsmittels erfasst. Abgefragt wird die Nutzung (ja/nein) von Stock/Gehhilfe, Gehwagen/ Rollator/Delta-Rad und Rollstuhl. Befragte, die mindestens eines dieser Hilfsmittel benutzen, gelten in der Analyse als in ihrer Mobilität eingeschränkt. Die Frage nach der Nutzung eines Mobilitätshilfsmittels ist in Bezug auf die Barrierefreiheit sinnvoll, weil diese Hilfsmittel selbst innerhalb der Wohnung beziehungsweise beim $\mathrm{Zu}$ gang zur Wohnung zu einem Hindernis werden können, wenn sie über Treppen, Schwellen oder durch schmale Türen bewegt werden müssen.

Analysen. Im Text und den Abbildungen werden jahres- und gruppenspezifische gewichtete Häufigkeiten oder prozentuale Häufigkeiten angegeben. Um Unterschiede zwischen Gruppen und/oder Jahren auf statistische Signifikanz $(p<, 05) \mathrm{zu}$ testen, wurden lineare, logistische und ordinale Regressionen berechnet, wobei für die Stratifizierungsvariablen Altersgruppe (40 bis 54 Jahre, 55 bis 69 Jahre und 70 bis 85 Jahre), Geschlecht und Region (West-/Ostdeutschland) kontrolliert wurde. Das genaue Vorgehen ist in Kapitel 2 beschrieben.

\subsection{Wohneigentum}

In der zweiten Lebenshälfte wohnt die Mehrheit der Bevölkerung in einer Wohnung oder einem Haus, das ihnen selbst gehört. Der Anteil der Personen mit Wohneigentum hat sich zwischen 1996 und 2014 von 57,6 auf 61,6 Prozent erhöht
(Abbildung 19-1). Nur noch ein Drittel der 40bis 85-Jährigen wohnt zur Miete (33,7 Prozent) und einige wenige wohnen mietfrei, zur Untermiete oder anderweitig geregelt in ihrer Wohneinheit (4,7 Prozent). 


\section{Ein steigender Anteil älterer Menschen wohnt in der eigenen Immobilie.}

Zwar ist die Wohneigentumsquote in den alten Bundesländern immer noch höher als in den neuen Bundesländern und bei den 55- bis 69-Jährigen höher als bei den beiden anderen Altersgruppen - durch die überdurchschnittliche Zunahme des Wohneigentums in Ostdeutschland und bei den 70- bis 85-Jährigen (Anstieg der Wohneigentümerquote auf 59,7 Prozent) haben sich die Unterschiede in den privaten Wohnfor- men zwischen Ost und West und zwischen den Altersgruppen jedoch verringert. Insgesamt ist nach einer deutlichen Zunahme des Wohneigentums bis zum Jahr 2008 in der Folgezeit eine Stagnation eingetreten, in der die Eigentümerquote nicht weiter gestiegen ist (vgl. Tabelle A 19-1 im Anhang). Die seit mehreren Jahren günstigen Finanzierungskonditionen, die relativ guten Arbeitsmarktbedingungen und das steigende Angebot an Kaufimmobilien lassen für die Zukunft aber eine weitere Zunahme des Wohnens in der eigenen Immobilie erwarten.

Abbildung 19-1: Wohneigentumsquote, gesamt, nach Alter und Landesteil, in den Jahren 1996 und 2014 (in Prozent)

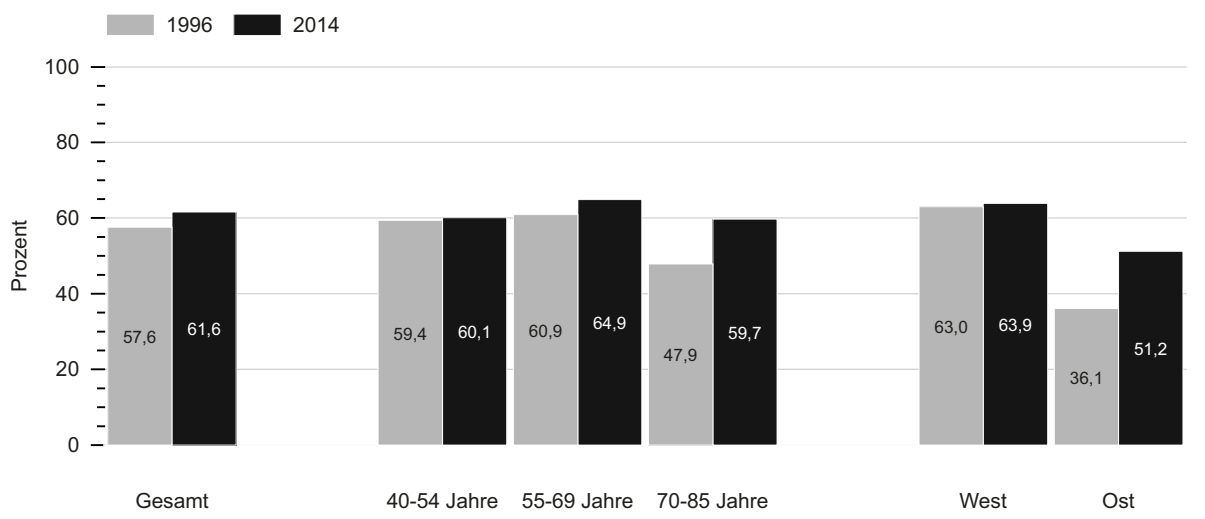

Quelle: DEAS $1996(n=4.838)$ und $2014(n=6.000)$, gewichtet, gerundete Angaben; $(p<, 05)$.

Signifikanter Anstieg für die Altersgruppen 55 bis 69 Jahre und 70 bis 85 Jahre und für beide Landesteile. Zunahme bei den 70- bis 85 -Jährigen und in Ostdeutschland signifikant höher als bei den anderen beiden Altersgruppen bzw. in Westdeutschland.

Mehr als drei Viertel (78,0 Prozent) der Wohneigentümer des Jahres 2014 haben ihre Wohnung oder ihr Haus gekauft, 22,0 Prozent haben die Immobilie durch eine Erbschaft, Schenkung oder anderweitige Eigentumsfeststellung erhalten. Im Vergleich zum Jahr 1996 erhöhte sich damit der Anteil des durch Kauf erworbenen Wohneigentums um 6,1 Prozentpunkte (ohne Abbildung). Es verwundert daher nicht, dass auch ein wachsender Anteil der Personen mit Wohneigentum Immobilienschulden hat und laufende Abzahlungsraten leisten muss.

\section{Der Anteil der Eigentümerhaushalte, die noch laufende Abzahlungen von Immobiliendarlehen leisten müssen, hat zugenommen.}

Im Jahr 2014 haben insgesamt 39,7 Prozent der Personen mit Wohneigentum für dieses ein Darlehen abzuzahlen, bei den 40 - bis 54-Jährigen sind es sogar 65,2 Prozent (vgl. Tabelle A 19-2 im Anhang). Je älter die Wohneigentümer sind, desto häufiger ist ihre Wohnung oder ihr Haus bereits schuldenfrei. Aber auch von den über 70-Jährigen des Jahres 2014 müssen noch 11,3 Prozent für das von ihnen erworbene Wohneigentum Abzahlungen leisten, deut- 
lich mehr als noch 2008 (7,0 Prozent). Etwas überraschend sinkt seit 2002 in Ostdeutschland der Anteil der Wohneigentümer, die noch ein Immobiliendarlehen bedienen müssen (2002: 41,2 Prozent; 2014: 35,9 Prozent). Möglicher- weise ist dies Folge einer verbesserten Ausstattung der ostdeutschen Käufer mit Eigenkapital und der wachsenden Zahl vollständiger Darlehenstilgungen der in den 1990er Jahren privat erworbenen Wohnimmobilien.

\subsection{Bewertung der Wohnsituation}

Die Erhebungen des DEAS zeigten in den vergangenen Jahren, dass die meisten Menschen in der zweiten Lebenshälfte ihre Wohnsituation positiv sehen. Daran hat sich auch im Jahr 2014 nichts geändert.

\section{Die eigene Wohnsituation wird im Jahr 2014 von einer großen Mehrheit der 40- bis 85-Jährigen als gut bewertet.}

89,7 Prozent der 40- bis 85 -Jährigen schätzen im Jahr 2014 ihre Wohnsituation als gut ein (vgl. Tabelle A 19-5 im Anhang). In der ersten DEASBefragung 1996 waren es noch 84,3 Prozent. Ältere sehen dabei ihre Wohnsituation signifikant häufiger positiv als Erwachsene im mittleren Erwachsenenalter, auch wenn die Unterschiede nur wenige Prozentpunkte betragen. Die 40- bis 54-Jährigen geben zu 88,0 Prozent, die 55- bis 69-Jährigen zu 90,1 Prozent und die Altersgruppe der 70- bis 85-Jährigen zu 92,0 Prozent eine positive Einschätzung ihrer Wohnsituation ab. Eine Erklärung für die über die Altersgruppen hinweg zunehmend positive Bewertung des eigenen Wohnens ist das Zufriedenheits- beziehungsweise Altersinvarianzparadox, das eine stabile positive Bewertung der Lebenssituation in relativer Unabhängigkeit von den objektiven Bedingungen beschreibt (Teti, Grittner, Kuhlmey, \& Blüher 2014; vgl. Kapitel 10). Eine hohe Wohnzufriedenheit sollte daher nicht darüber hinweg täuschen, dass trotzdem ein Bedarf an verbesserten Wohnbedingungen für Ältere existieren kann.

Auch im Vergleich von West- und Ostdeutschland zeigt sich die Wohnzufriedenheit auf einem stabil hohen Niveau. In Ostdeutsch- land lässt sich ein Aufholprozess seit 1996 beobachten mit einer hohen Dynamik in den 1990er Jahren und einem langsameren Anwachsen seit 2002 (Abbildung 19-2). Nachdem es bis 2008 noch eine signifikante Differenz in der Bewertung der Wohnsituation von West- und Ostdeutschen gab, ist diese Differenz im Jahr 2014 zum ersten Mal nicht mehr zu beobachten. Ostdeutsche in der zweiten Lebenshälfte sehen im Jahr 2014 ihre Wohnsituation etwa gleich positiv wie die Westdeutschen.

\section{Auch in einfachen Wohnlagen bezeichnen die Bewohnerinnen und Bewohner ihre Wohnsituation mehrheitlich als gut.}

Bemerkenswert ist, dass auch in den von den Interviewenden als einfach eingeschätzten Wohnlagen die Bewohnerinnen und Bewohner ihre Wohnsituation mehrheitlich als gut bezeichnen, auch wenn der Anteil positiver Bewertungen deutlich geringer ist als in gehobenen oder mittleren Wohnlagen (Abbildung 19-3). 96,2 Prozent der 40- bis 85-Jährigen in gehobenen Wohnlagen bezeichnen ihre Wohnsituation als gut, 87,9 Prozent der Bewohner mittlerer Wohnlagen und immerhin noch 68,1 Prozent der Bewohnerinnen und Bewohner einfacher Wohnlagen tun dies. Für Menschen in der zweiten Lebenshälfte spielt offenbar für die Bewertung ihrer Wohnsituation nicht in erster Linie der bauliche Zustand der Wohnung und des Wohnumfelds eine Rolle, sondern auch in hohem Maße ihre Verbundenheit mit der Wohnung, dem Quartier und der Nachbarschaft (vgl. dazu Kapitel 20 und 21). 
Abbildung 19-2: Anteile der Personen, die ihre Wohnsituation als ,gut' bewerten, nach Landesteil, in den Jahren 1996, 2002, 2008 und 2014 (in Prozent)

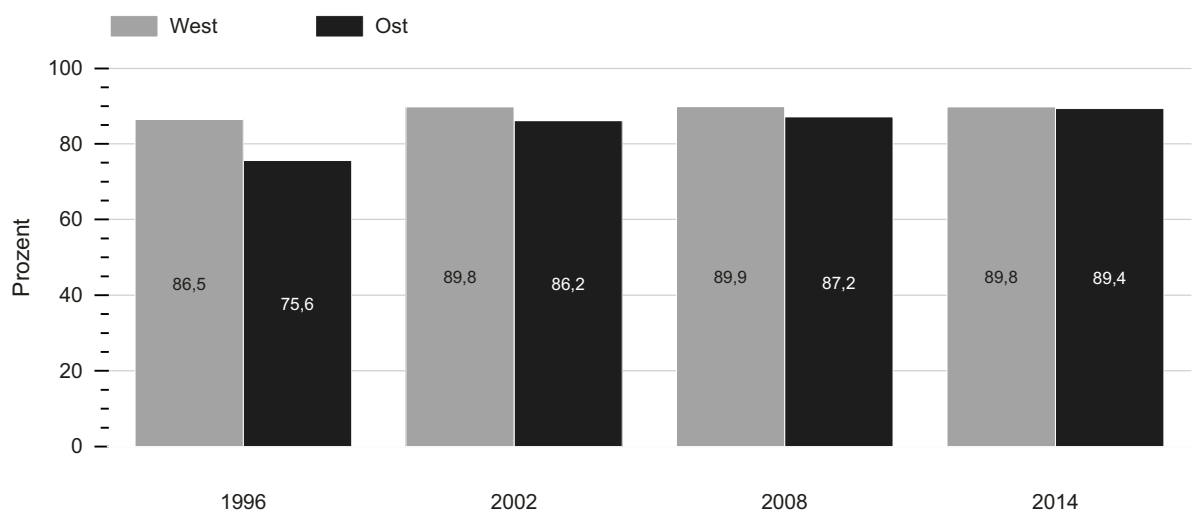

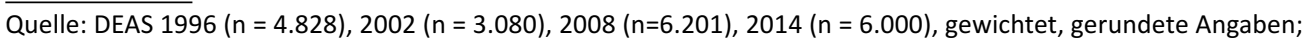
$(p<, 05)$

Signifikanter Unterschied zwischen West- und Ostdeutschland in den Jahren 1996, 2002, 2008. Kein signifikanter Unterschied im Jahr 2014.

Abbildung 19-3: Anteile der Personen, die ihre Wohnsituation als ,gut' bewerten, nach Wohnlage, im Jahr 2014 (in Prozent)

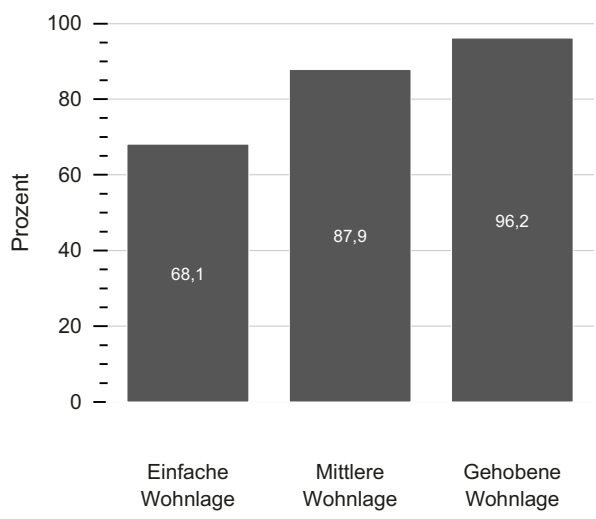

Quelle: DEAS 2014 ( $n=5.954)$, gewichtete, gerundete Angaben; $(p<, 05)$.

Signifikante Unterschiede in beiden Kategorien zwischen allen Wohnlagen.

\subsection{Wohnkosten}

Die monatlichen Wohnkosten können unterschiedlich hoch sein. Unter anderem hängen sie davon ab, ob es sich um eine Mietwohnung oder um Wohneigentum handelt und ob dieses bereits schuldenfrei ist. Im Jahr 2014 beträgt die von den Haushalten der 40- bis 85-Jährigen zu begleichende durchschnittliche Monatsmiete einschließlich Nebenkosten 703 Euro. Gegen- 
über 1996 hat sich die Miete um 57 Prozent erhöht. Da die Einkommen nicht im selben Maße gestiegen sind (vgl. Kapitel 6), erhöhte sich die Mietbelastung.

\section{Die monatliche Wohnkostenbelastung in Prozent des Einkommens hat zwischen 1996 und 2014 zugenommen.}

Im Jahr 2014 müssen Mieterhaushalte 35,1 Prozent ihres monatlichen Nettoeinkommens für die Miete und Nebenkosten ausgeben, im Jahr 1996 waren es erst 27,9 Prozent (Abbildung 19-4). Eigentümerhaushalte, die noch Darlehensraten für ihre Immobilie zu leisten ha- ben, wenden hierfür zuzüglich der monatlichen Nebenkosten im Durchschnitt 1.091 Euro auf. Dies sind 32,8 Prozent ihres Haushaltsnettoeinkommens. Ihre Wohnkostenbelastung ist damit erstmals etwas geringer als die der Mieterhaushalte. Dazu beigetragen haben der gebremste Anstieg der nominalen Wohnkosten nach 2008 und ihr im Vergleich zu Mieterhaushalten höherer Einkommenszuwachs. Die niedrigsten Wohnkosten absolut und in Relation zu ihrem Einkommen haben Eigentümerhaushalte, deren Immobilie schuldenfrei ist. Sie müssen nur 16 Prozent ihres Haushaltseinkommens für die monatlichen Wohnkosten aufwenden, da sie keine Wohndarlehensraten (mehr) aufbringen müssen.

Abbildung 19-4: Anteil monatlicher Wohnkosten am Haushaltseinkommen nach Wohnform, in den Jahren 1996, 2002, 2008 und 2014 (in Prozent)

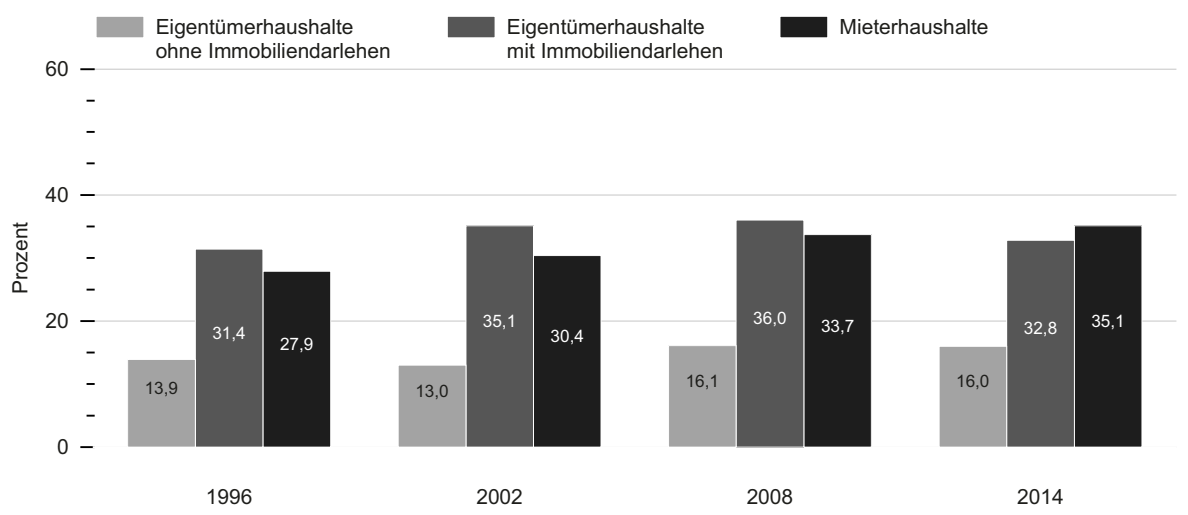

$\overline{\text { Quelle: DEAS } 1996}(n=3.415), 2002(n=2.023), 2008(n=4.416), 2014(n=4.655)$, gewichtet, gerundete Angaben; $(p<, 05)$.

Signifikante Unterschiede zwischen 1996 und 2014 für alle drei Kategorien. Signifikante Unterschiede zwischen allen Kategorien zu allen Zeitpunkten, ausgenommen die Differenz zwischen Mieterhaushalten und Eigentümerhaushalten mit Immobiliendarlehen im Jahr 2014.

Bei Mieterhaushalten steigt mit dem Alter die Wohnkostenbelastung, was in Verbindung mit der Absenkung des Haushaltseinkommens beim Übergang in den Ruhestand und nach einer Verwitwung zu sehen ist. Die im Jahr 2014 zur Miete wohnenden 40- bis 54-Jährigen geben 33,1 Prozent ihres Haushaltseinkommens für Miete und Nebenkosten aus, die 70- bis 85-Jährigen müssen dafür 37,8 Prozent einsetzen (Abbildung 19-5). Über 70-jährige alleinlebende
Mieterinnen müssen sogar 45,2 Prozent ihres Einkommens für die Wohnkosten aufwenden (ohne Abbildung). Bei Eigentümerhaushalten ist der Alterszusammenhang umgekehrt. Bei ihnen verringert sich mit steigendem Alter die Wohnkostenbelastung, weil ältere Wohneigentümerinnnen und -eigentümer häufig keine Immobilienschulden (mehr) haben. Dann fallen nur noch die üblichen Nebenkosten an. 
Für Eigentümer- und Mieterhaushalte zeigt sich auch ein Zusammenhang der Wohnkostenbelastung mit der Wohnlage. ${ }^{2}$ Haushalte in einfachen Wohnlagen müssen einen höheren Anteil ihres Einkommens für die Wohnkosten aufwenden als Haushalte in mittleren und gehobenen Wohnlagen (Abbildung 19-5). Verantwortlich dafür ist das niedrigere Durchschnittseinkommen der in einfachen Wohnlagen lebenden Haushalte. ${ }^{3}$ Die Ost-West-Unterschiede der
Mietbelastung haben sich zwischen 1996 und 2014 aufgelöst. Während im Jahr 1996 ostdeutsche Mieterhaushalte mit 24,2 Prozent einen deutlich geringeren Anteil ihres Haushaltseinkommens für die Miete plus Nebenkosten aufwenden mussten als westdeutsche Mieterhaushalte (29,7 Prozent), betrug die Mietbelastung im Jahr 2014 in beiden Landesteilen 35 Prozent (Ost: 35,1 Prozent, West: 35,0 Prozent) (siehe Tabelle A 19-3 im Anhang).

2 Die Einstufung der Wohnlage beruht auf Angaben der Interviewerinnen und Interviewer (siehe Abschnitt 19.2).

3 Das im DEAS für 2014 ermittelte durchschnittliche Monatseinkommen der in einfachen Wohnla-

gen lebenden Mieterhaushalte liegt 44 Prozent unter dem Einkommen der Mieterhaushalte in gehobenen Wohnlagen (ohne Abbildung).

Abbildung 19-5: Anteil monatlicher Wohnkosten am Haushaltseinkommen, nach Alter und Wohnlage, im Jahr 2014 (in Prozent)

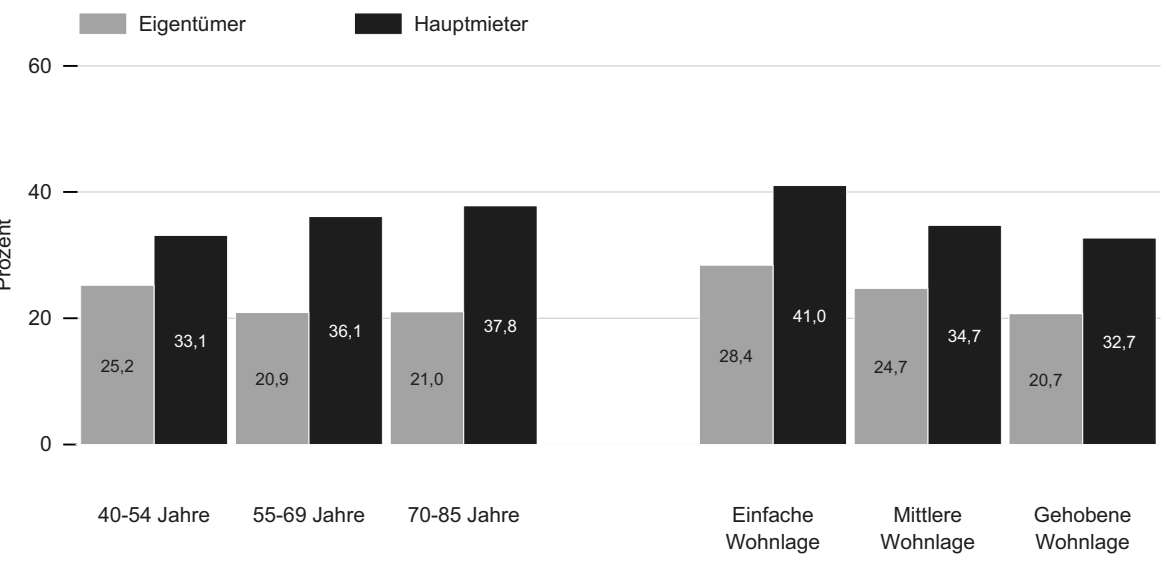

Quelle: DEAS 2014 ( $n=4.655$ für die Altersgruppen, $n=4.625$ für die Wohnlagen), gewichtet, gerundete Angaben; $(p<, 05)$.

Eigentümer: Signifikante Unterschiede nur zwischen 40- bis 54-Jährigen und den 55- bis 69-Jährigen bzw. 70- bis 85-Jährigen. Signifikante Unterschiede zwischen allen Wohnlagen. Hauptmieter: Signifikante Unterschiede nur zwischen 40- bis 54-Jährigen und den 55- bis 69-Jährigen bzw. 70- bis 85-Jährigen. Signifikanter Unterschied zwischen einfacher Wohnlage und mittlerer bzw. gehobener Wohnlage.

Insgesamt ist das Wohnen teurer geworden. Insbesondere Personen, die zur Miete wohnen, müssen einen wachsenden Anteil ihres Einkommens für die Miete und die Nebenkosten aufwenden. Die steigende Quote von Menschen mit Wohneigentum wurde erkauft durch einen wachsenden Anteil von Haushalten mit einer relativ hohen monatlichen Belastung durch das Abzahlen von Immobiliendarlehen. Gelingt es Personen mit Wohneigentum allerdings, ihr Wohndarlehen bis zum Beginn des Ruhestands vollständig zu tilgen und fortan in der abbezahlten Immobilie zu wohnen, reduziert dies ihre laufenden Wohnkosten im Alter erheblich. 


\subsection{Barrierereduzierte Wohnungen}

Gerade im hohen Alter nehmen Mobilitätseinschränkungen immer mehr zu (vgl. Kapitel 8). Um auch mit solchen Einschränkungen weiterhin gut in der eigenen Wohnung leben zu können, kommt es darauf an, dass die Wohnung möglichst wenige Barrieren hat.

\section{Barrierereduzierte Wohnungen sind im Jahr 2014 wenig verbreitet.}

Es gibt nicht viele Menschen, die in einer barrierereduzierten Wohnung leben. Legt man die im Abschnitt ,Datengrundlagen ' beschriebenen Kriterien zugrunde, so leben von den 40 - bis 85-Jährigen nur 2,9 Prozent in einer solchen Wohnung. Dieser Anteil ist erwartungsgemäß in der ältesten Altersgruppe am größten, da in dieser Altersgruppe die gesundheitlichen Einschränkungen und damit der Bedarf an Wohnbedingungen ohne Barrieren besonders hoch sind. Von den 70- bis 85-Jährigen wohnen 5,6 Prozent unter barrierereduzierten Bedingungen (Abbildung 19-6). In Ostdeutschland gibt es ei- nen etwas höheren Anteil barrierereduzierter Wohnungen $(4,1$ Prozent) als in Westdeutschland (2,6 Prozent). Eine Erklärung dafür kann sein, dass seit den 1990er Jahren in Ostdeutschland zahlreiche Wohnungen modernisiert beziehungsweise neu gebaut wurden, die zu einem höheren Anteil als in Westdeutschland den Standards der Barrierefreiheit genügen.

Was bedeutet dieser Befund? Ob eine Wohnung den Wohnbedürfnissen ihrer Bewohnerinnen und Bewohner entspricht, hängt stark von deren Gesundheitszustand und auch von ihren individuellen Wohnpräferenzen ab. Nicht jeder Mensch im höheren Alter hat größere funktionale Einschränkungen (vgl. Kapitel 8). Viele Ältere richten sich in den vorhandenen Wohnbedingungen ein, auch wenn diese nicht optimal an ihre Bedürfnisse angepasst sind. Dennoch kann angesichts der sehr geringen Anteilswerte an barrierereduzierten Wohnungen davon ausgegangen werden, dass ein größerer Nachholbedarf an diesen Wohnungen besteht, der in den kommenden Jahren infolge der demografischen Alterung noch wachsen dürfte.

Abbildung 19-6: Anteile der in einer barrierereduzierten Wohnung lebenden Menschen, gesamt, nach Alter, Geschlecht und Landesteil, im Jahr 2014 (in Prozent)

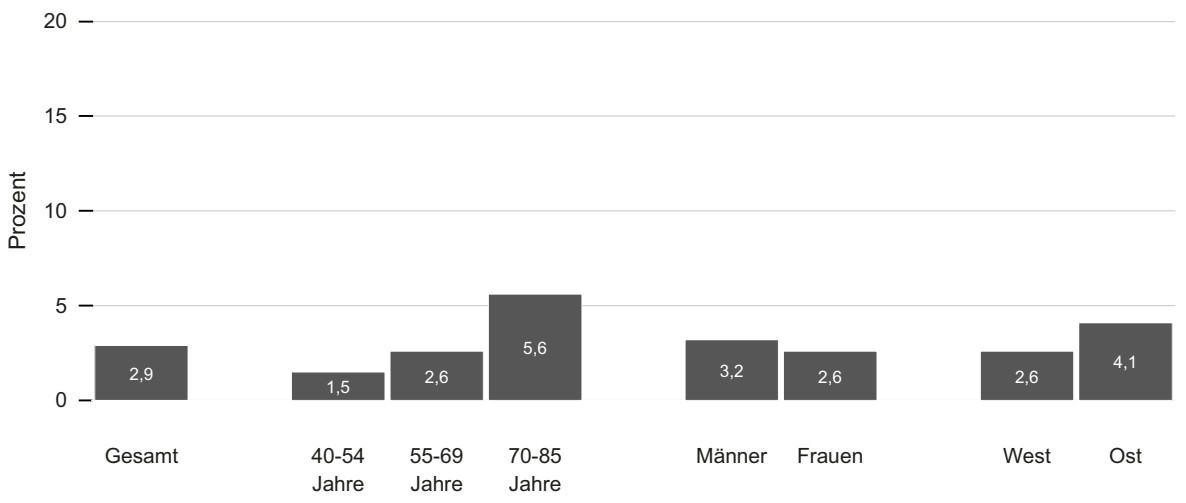

Quelle: DEAS 2014 ( $n=4.226)$, gewichtet, gerundete Angaben; $(p<, 05)$.

Signifikante Alters- und Landesteilunterschiede. Keine signifikanten Geschlechtsunterschiede. 


\section{Auch Ältere mit Mobilitätseinschränkungen haben überwiegend keine barrierereduzierte Wohnung.}

Wie leben Menschen, die aufgrund von körperlichen Einschränkungen, insbesondere der Mobilität, auf barrierereduzierte Wohnbedingungen angewiesen sind, um ihren Alltag gut bewältigen zu können? Auch von ihnen ist nur eine Minderheit mit entsprechenden Wohnbedingungen versorgt. Von denjenigen, die auf die Nutzung einer Gehhilfe, eines Rollators oder eines Rollstuhls angewiesen sind, haben lediglich 6,9 Prozent eine barrierereduzierte Wohnung. Abbildung 19-7 zeigt, dass diese Personen zum überwiegenden Teil in Wohnungen leben, die keinen barrierefreien Zugang haben beziehungsweise die im Inneren nicht barrierefrei sind. Nur ein knappes Viertel der in ihrer Mobilität Eingeschränkten kommt ohne Barrieren in die eigene Wohnung (23,9 Prozent), der Un- terschied zur Gruppe der nicht Mobilitätseingeschränkten ist nicht signifikant. Mobilitätseinschränkungen hängen also nicht mit mehr barrierereduzierten Zugängen zur Wohnung zusammen. 13,5 Prozent der in ihrer Mobilität Eingeschränkten haben eine Wohnung, die im Inneren barrierereduziert ist (zu den Kriterien siehe Abschnitt 19.2). Hier gibt es einen signifikanten Unterschied zur Vergleichsgruppe der nicht Mobilitätseingeschränkten (6,6 Prozent). Das heißt, wenn Personen mit Gehhilfe, Rollator oder Rollstuhl ihre Wohnbedingungen im Sinne der Barrierefreiheit anpassen, dann erfolgt das eher durch Umbaumaßnahmen innerhalb der Wohnung. Umrüstungsmaßnahmen, die für den Zugang zur Wohnung Barrieren beseitigen, betreffen die Architektur des gesamten Hauses und sind offenbar schwieriger zu bewerkstelligen. Das bedeutet, dass Barrierefreiheit des Wohnungszugangs beim Neubau von Häusern beziehungsweise bei umfassenden Sanierungen von vornherein mitgedacht werden muss.

\section{Abbildung 19-7: Barrieren in den Wohnungen von mobilitätseingeschränkten und nicht mobilitäts-} eingeschränkten Menschen, im Jahr 2014 (in Prozent)

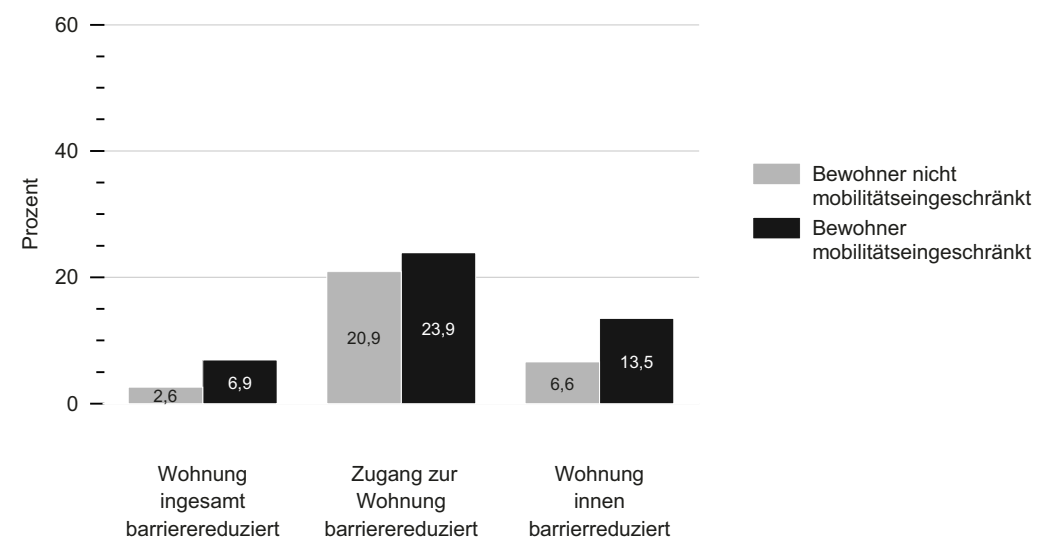

Quelle: DEAS 2014 ( $n=3.910)$, gewichtet, gerundete Angaben; $(p<, 05)$.

Mobilitätseingeschränkt heißt Nutzung von Gehhilfen, Rollatoren, Rollstühlen o. ä.

Signifikante Unterschiede zwischen mobilitätseingeschränkten und nicht mobilitätseingeschränkten Bewohnerinnen und Bewohnern bei ,Wohnung insgesamt barrierereduziert' und bei ,Wohnung innen barrierereduziert'. Keine signifikanten Unterschiede bei ,Zugang zur Wohnung barrierereduziert'.

Zum Wohnkomfort für Menschen mit funktionalen Beeinträchtigungen gehört auch die sanitäre Ausstattung, von der einige Merkmale im DEAS erfasst werden. In der Gruppe von
Personen, die auf Mobilitätshilfsmittel angewiesen sind, verfügen 74,8 Prozent über ein $\mathrm{Bad}$ mit Badewanne, 38,6 Prozent über eine Dusche mit Duschtasse und nur 26,6 Prozent über eine 
bodengleiche Dusche. Außerdem verfügen von den Nutzern eines Mobilitätshilfsmittels 8,5 Prozent über einen Notruf in der Wohnung beziehungsweise im Haus (im Vergleich zu nur 1,3
Prozent derjenigen, die keine Mobilitätshilfen nutzen) und 6,5 Prozent über einen Treppenlift (im Vergleich zu 1,9 Prozent derjenigen, die keine Mobilitätshilfen nutzen) (ohne Abbildung).

\subsection{Diskussion und Implikationen}

Ältere Menschen leben überwiegend in einem privaten Haushalt. Die meisten von ihnen haben den Wunsch, bis in das hohe Alter ein selbstbestimmtes Leben in den eigenen vier Wänden zu führen. Mit steigendem Alter und den damit häufig verbundenen funktionalen Einschränkungen verändern sich die Anforderungen an das Wohnen - ein Aspekt, der in den letzten Jahren verstärkt in das öffentliche Bewusstsein gelangt ist. Die Befunde des DEAS veranschaulichen einige Aspekte des Wohnens im Alter.

Die Daten des DEAS zeigen, dass ältere Menschen ihre Wohnsituation mehrheitlich als gut beurteilen. Sie fühlen sich mit ihrer Wohnung, dem Wohnumfeld und der Nachbarschaft verbunden. Der an sich positive Umstand der Bindung an die Wohnung kann sich aber auch nachteilig auswirken, wenn jahrzehntelanges Wohnen das Ausharren in nicht mehr den Bedürfnissen entsprechenden Wohnbedingungen bedeutet. Hier finden sich Ansatzpunkte, um durch Wohnraumanpassung altersadäquate Wohnbedingungen zu fördern. Ein anderer Ansatz ist es, älteren Menschen den Umzug in eine andere Wohnung zu erleichtern. Als hemmender Faktor für die Umzugsbereitschaft erweist sich die Mietenentwicklung, gerade in größeren Städten und begehrten Wohnlagen. Sie behindert Wohnmobilität, indem ein Umzug in eine kleinere Wohnung mit altersangepassten Wohnbedingungen häufig mit höheren Wohnkosten erkauft werden muss.

Die Wohnkosten sind gestiegen und stellen im Alter eine überdurchschnittliche finanzielle Belastung dar. Ein wachsender Anteil des Einkommens muss für die Wohnkosten aufgewendet werden. Bei niedrigem Einkommen ist der finanzielle Spielraum dadurch erheblich eingeschränkt. Ein wachsender Anteil älterer
Menschen wohnt im Alter in der eigenen Immobilie. Wohneigentumsbildung führt allerdings nicht selbstverständlich zu einer finanziellen Entlastung im Alter. Teilweise sind auch nach Erreichen des Rentenalters noch Immobilienschulden abzubezahlen und die laufenden Kosten und Instandhaltungsaufwendungen sind - besonders bei größerem und älterem Wohneigentum - nicht zu unterschätzen. Auch die Möglichkeiten einer gewinnbringenden Veräußerung als finanzielles Polster im Alter werden voraussichtlich nicht überall gegeben sein, insbesondere nicht in strukturschwachen, von Abwanderung betroffenen Regionen, von denen viele in Ostdeutschland liegen.

Eine Wohnung, deren bauliche Ausstattung auch für Personen mit gesundheitlichen Einschränkungen einen funktionierenden Alltag erleichtert, kann die Lebensphase im gewohnten Umfeld verlängern und so zum Wohlbefinden Älterer beitragen. Die Ergebnisse zeigen, dass nur ein geringer Teil der Älteren in barrierereduzierten Wohnungen lebt. Zwischen Bedarf und Angebot an derartigen Wohnungen klafft bereits heute eine Lücke und für die kommenden Jahre ist als Folge der demografischen Alterung mit einer weiter erhöhten Nachfrage zu rechnen. Es gibt mittlerweile zahlreiche Programme und Initiativen, die barrierereduziertes Umbauen und technikunterstütztes Wohnen fördern. Durch Neubauten und Gebäudesanierungen sollte der Anteil barrierereduzierter Wohnungen am Wohnungsbestand künftig erhöht werden. Der Ausbau von Wohnberatungsstellen für Ältere kann die Möglichkeiten des Wohnungsumbaus im Sinne von Barrierefreiheit für mehr Menschen aufzeigen und dazu beitragen, den Anteil barrierereduzierten Wohnens zu erhöhen (vgl. auch Kapitel 20). Glei- 
ches gilt für die Entwicklung und Verbreitung technikbasierter Assistenzsysteme im Wohnbereich. Nicht zu unterschätzen ist auch der ökonomische Effekt, der gerade in Haushalten mit Pflegebedürftigen entstehen könnte: Auch sie sind mit barrierereduzierten Wohnungen unterversorgt. Bei einer besseren Versorgung mit adäquatem Wohnraum könnte der Heimeintritt bei einem Teil dieser Pflegebedürftigen hinausgezögert und so eine erhebliche Kostenersparnis erzielt werden. Schätzungen sprechen von 5,2 Milliarden Euro jährlich (Bundesinstitut für Bau- Stadt- und Raumforschung (BBSR) 2014).

\section{Literatur}

Bundesinstitut für Bau-, Stadt- und Raumforschung (2012). Raumabgrenzungen und Raumtypen des BBSR [Analysen Bau. Stadt. Raum 6]. Bonn: Bundesinstitut für Bau-, Stadt- und Raumforschung.

BertelsmannStiftung (Hrsg.) (2013). Wohnungsangebot für arme Familien in Großstädten. Bielefeld: BertelsmannStiftung.

Bundesinstitut für Bau-, Stadt- und Raumforschung (2014). Potenzialanalyse altersgerechte Wohnungsanpassung. Bonn: Bundesinstitut für Bau-, Stadt- und Raumforschung.

Bundesministerium für Verkehr, Bau und Stadtentwicklung (BMVBS) (2011). Wohnen im Alter - Marktprozesse und wohnungspolitischer Handlungsbedarf. Forschungen, 147. Berlin: Bundesministerium für Verkehr, Bau und Stadtentwicklung.

Engstler, H., Menning, S., Hoffmann, E., \& Tesch-Römer, C. (2004). Die Zeitverwendung älterer Menschen. In: Statistisches Bundesamt (Hrsg.) Alltag in Deutschland - Analysen zur Zeitverwendung (S. 216-246). Stuttgart: Metzler-Poeschel.

Frick, J., \& Droß, P. J. (2011). Wohnverhältnisse und Wohnkosten. In: Statistisches Bundesamt \& Wissenschaftzentrum Berlin für Sozialforschung (Hrsg.) Datenreport 2011 (Bd. 1, S. 207-214). Bonn: Bundeszentrale für politische Bildung.

Kaltenbrunner, R., \& Waltersbacher, M. (2014). Wohnsituation in Deutschland. Aus Politik und Zeitgeschichte, 64(20-21), 3-12.

Kocka, J., \& Staudinger, U. (2009). Gewonnene Jahre. Empfehlungen der Akademiengruppe Altern in Deutschland [Altern in Deutschland 9]. Stuttgart: Wissenschaftliche Verlagsgesellschaft.

Kuratorium Deutsche Altershilfe \& Wüstenrot Stiftung (2014). Wohnatlas. Rahmenbedingungen der Bundesländer beim Wohnen im Alter. Teil 1: Bestandsanalyse und Praxisbeispiele. Köln: Kuratorium Deutsche Altershilfe.

Motel-Klingebiel, A., Künemund, H., \& Bode, C. (2005). Wohnen und Wohnumfeld. In: M. Kohli \& H. Künemund (Hrsg.) Die zweite Lebenshälfte. Gesellschaftliche Lage und Partizipation im Spiegel des Alters-Survey (S. 125-175). Wiesbaden: VS Verlag für Sozialwissenschaften.

Oswald, F., Jopp, D., Rott, C., \& Wahl, H. W. (2011). Is aging in place a resource for or risk to life satisfaction? Gerontologist, 51(2), 238-250. doi: 10.1093/geront/ gnq096.

Pestel Institut (2012). Bedarf an Sozialwohnungen in Deutschland. Untersuchung im Auftrag der Wohnungsbau Initiative. Hannover: Pestel Institut.

Priem, M., \& Schupp, J. (2014). Alle zufrieden: Lebensverhältnisse in Deutschland. DIW Wochenbericht, 81(40), 1001-1008.

Rothgang, H., Kalwitzki, T., Müller, R., Runte, R., \& Unger, R. (2015). BARMER GEK PFLEGEREPORT 2015. Schwerpunktthema: Pflegen zu Hause. Siegburg: Asgard-Verlagsservice.

Statistisches Bundesamt (2015). Mikrozensus 2014 Sonderauswertungen für das Deutsche Zentrum für Altersfragen. Wiesbaden: Statistisches Bundesamt.

Statistisches Bundesamt (2012). Wohnsituation der Haushalte 2010. Wiesbaden: Statistisches Bundesamt.

Teti, A., Grittner, U., Kuhlmey, A., \& Blüher, S. (2014). Wohnmobilität im Alter. Zeitschrift für Gerontologie und Geriatrie, 47(4), 320-328. doi: 10.1007/s00391013-0538-0.

Open Access Dieses Kapitel wird unter der Creative Commons Namensnennung 2.5 International Lizenz (http://creativecommons.org/licenses/by/2.5/deed.de) veröffentlicht, welche die Nutzung, Vervielfältigung, Bearbeitung, Verbreitung und Wiedergabe in jeglichem Medium und Format erlaubt, sofern Sie den/die ursprünglichen Autor(en) und die Quelle ordnungsgemäß nennen, einen Link zur Creative Commons Lizenz beifügen und angeben, ob Änderungen vorgenommen wurden.

Die in diesem Kapitel enthaltenen Bilder und sonstiges Drittmaterial unterliegen ebenfalls der genannten Creative Commons Lizenz, sofern sich aus der Abbildungslegende nichts anderes ergibt. Sofern das betreffende Material nicht unter der genannten Creative Commons Lizenz steht und die betreffende Handlung nicht nach gesetzlichen Vorschriften erlaubt ist, ist für die oben aufgeführten Weiterverwendungen des Materials die Einwilligung des jeweiligen Rechteinhabers einzuholen. 